\title{
Identification of Patients Affected by Mitral Valve Prolapse with Severe Regurgitation: A Multivariable Regression Model
}

\author{
Paola Songia, ${ }^{1,2}$ Benedetta Porro, ${ }^{1}$ Mattia Chiesa, ${ }^{1}$ Veronika Myasoedova, \\ Francesco Alamanni, ${ }^{1,3}$ Elena Tremoli, ${ }^{1}$ and Paolo Poggio ${ }^{1}$ \\ ${ }^{1}$ Centro Cardiologico Monzino IRCCS, Milan, Italy \\ ${ }^{2}$ Dipartimento di Scienze Farmacologiche e Biomolecolari, Università degli Studi di Milano, Milan, Italy \\ ${ }^{3}$ Dipartimento di Scienze Cliniche e di Comunità, Università degli Studi di Milano, Milan, Italy \\ Correspondence should be addressed to Paolo Poggio; paolo.poggio@ccfm.it
}

Received 8 September 2016; Revised 30 December 2016; Accepted 11 January 2017; Published 2 February 2017

Academic Editor: Cecilia Zazueta

Copyright (C) 2017 Paola Songia et al. This is an open access article distributed under the Creative Commons Attribution License, which permits unrestricted use, distribution, and reproduction in any medium, provided the original work is properly cited.

\begin{abstract}
Background. Mitral valve prolapse (MVP) is the most common cause of severe mitral regurgitation. Besides echocardiography, up to now there are no reliable biomarkers available for the identification of this pathology. We aim to generate a predictive model, based on circulating biomarkers, able to identify MVP patients with the highest accuracy. Methods. We analysed 43 patients who underwent mitral valve repair due to MVP and compared to 29 matched controls. We assessed the oxidative stress status measuring the oxidized and the reduced form of glutathione by liquid chromatography-tandem mass spectrometry method. Osteoprotegerin (OPG) plasma levels were measured by an enzyme-linked immunosorbent assay. The combination of these biochemical variables was used to implement several logistic regression models. Results. Oxidative stress levels and OPG concentrations were significantly higher in patients compared to control subjects $(0.116 \pm 0.007$ versus $0.053 \pm 0.013$ and $1748 \pm 100.2$ versus $1109 \pm 45.3 \mathrm{pg} / \mathrm{mL}$, respectively; $p<0.0001)$. The best regression model was able to correctly classify 62 samples out of 72 with accuracy in terms of area under the curve of 0.92 . Conclusions. To the best of our knowledge, this is the first study to show a strong association between OPG and oxidative stress status in patients affected by MVP with severe regurgitation.
\end{abstract}

\section{Introduction}

Myxomatous mitral valve prolapse (MVP) is the most common indication for mitral valve surgery due to severe mitral regurgitation (MR). The prevalence of MVP is estimated at 2$3 \%$ with approximately 144 million people affected worldwide [1]. Echocardiographically, MVP is defined as a single or bileaflet prolapse, at least $2 \mathrm{~mm}$ beyond the long-axis annular plane, while the assessment of valve regurgitation takes into account the effective regurgitant orifice area (EROA) [2]. Interestingly, the pathology is equally distributed between men and women [3] and patients with prolapse have significantly lower body-mass index (BMI) and waist-to-hip ratio than those without prolapse [1].

Despite the pathology was first described in the late 1800s [3], no major risk factor has been identified yet [4] and the triggering mechanisms of MVP are not fully understood. In this context, a very recent study by Deroyer et al. [5] showed a negative association between severity of MR and high-density lipoproteins (HDL) levels. Interestingly, HDL concentrations and levels of Apo-Al, the major protein component of HDL, which participates in the reverse cholesterol transport, decreased according to the severity of MR. In addition, MVP patients showed an alteration in the antioxidant defence systems and an increase in lipid peroxidation markers [6].

Given the background, we investigated Osteoprotegerin (OPG), a well-known protein linked to oxidative stress status [7] and to endothelial mesenchymal transition on endothelial cells isolated from MVP patients [8]. OPG is a secretory glycoprotein of the tumour necrosis factor receptor superfamily involved in calcification, apoptosis, proliferation, and migration processes [9]. It possesses high affinity to receptor activator of nuclear factor-kb (RANKL), to tumour necrosis factor-related apoptosis-inducing ligand (TRAIL), 
TABle 1: Patient demographics.

\begin{tabular}{|c|c|c|c|}
\hline & Control $(N=29)$ & $\operatorname{MVP}(N=43)$ & $p$ value \\
\hline \multicolumn{4}{|l|}{ Variable } \\
\hline Age (years) & $57.3[53.2,61.4]$ & $60.1[56.8,63.5]$ & 0.291 \\
\hline Sex (male) & $19(65.5 \%)$ & $29(67.4 \%)$ & 0.865 \\
\hline Diabetes & $3(10.3 \%)$ & $3(7.0 \%)$ & 0.685 \\
\hline Hypertension & $14(48.3 \%)$ & $16(37.2 \%)$ & 0.660 \\
\hline Hypercholesterolemia & $14(48.3 \%)$ & $25(58.1 \%)$ & 0.688 \\
\hline Smokers & $6(20.7 \%)$ & $5(11.6 \%)$ & 0.514 \\
\hline BMI & $27.4 \pm 0.76$ & $24.8 \pm 0.43$ & 0.005 \\
\hline Total cholesterol (mg/dL) & $216.1 \pm 7.4$ & $215.2 \pm 6.6$ & 0.973 \\
\hline Triglycerides (mg/dL) & $111.2 \pm 9.0$ & $110.3 \pm 6.7$ & 0.690 \\
\hline $\mathrm{HDL}(\mathrm{mg} / \mathrm{dL})$ & $55.8 \pm 3.2$ & $51.3 \pm 1.9$ & 0.249 \\
\hline $\mathrm{LDL}(\mathrm{mg} / \mathrm{dL})$ & $138.0 \pm 7.1$ & $132.4 \pm 6.5$ & 0.658 \\
\hline NYHA class & & & 0.001 \\
\hline I & $29(100 \%)$ & $19(44.2 \%)$ & \\
\hline II & - & $17(39.5 \%)$ & \\
\hline III & - & $7(16.3)$ & \\
\hline IV & - & - & \\
\hline \multicolumn{4}{|l|}{ Drug therapies } \\
\hline Antiplatelets (\%) & $1(3 \%)$ & $2(5 \%)$ & 1.00 \\
\hline Angiotensin receptor blockers (\%) & $4(14 \%)$ & $3(7 \%)$ & 0.429 \\
\hline Converting enzyme inhibitors (\%) & $5(17 \%)$ & $14(32 \%)$ & 0.295 \\
\hline Calcium channel blockers (\%) & $3(10 \%)$ & $3(7 \%)$ & 0.679 \\
\hline Beta-blockers (\%) & $1(3 \%)$ & $16(37 \%)$ & 0.007 \\
\hline Nitrates (\%) & $0(0 \%)$ & $1(2 \%)$ & 1.00 \\
\hline Statins (\%) & $6(21 \%)$ & $6(14 \%)$ & 0.527 \\
\hline
\end{tabular}

BMI: body mass index; HDL: high-density lipoprotein; LDL: low-density lipoprotein; NYHA: New York Heart Association. [minimum, maximum]; (percentage); mean \pm standard error.

and to syndecan family receptors (SDC). This molecule has been associated with cardiometabolic disorders [9], as well as increased cardiovascular and overall mortality $[10,11]$. In addition, it has been shown that transforming growth factor beta (TGF- $\beta$ ), a well-known player in myxomatous MVP pathogenesis [12], increases oxidative stress status [13] as well as OPG production and secretion directly stimulating OPG promoter activity [14].

In this study, we aim to generate a predictive model able to identify MVP patients with the highest accuracy with the combination of biochemical parameters easily quantifiable.

\section{Patients and Methods}

2.1. Patient Demographics. This observational study was approved by the Institutional Review Board and by the Ethical Committee of Centro Cardiologico Monzino (CCM), IRCCS. The investigation conformed to the principles outlined in the Declaration of Helsinki (1964).

A total of 51 consecutive patients that underwent mitral valve repair, at CCM, due to MVP were enrolled. Based on exclusion criteria (presence of bicuspid aortic valve, premature menopause, and/or osteoporosis, prior aortic or mitral valve surgery, rheumatic heart disease, endocarditis, active malignancy, chronic liver failure, calcium regulation disorders, and chronic or acute inflammatory states), we selected and analysed 43 patients matched by age, sex, diabetes, hypertension, hypercholesterolemia, and smoking habits with 29 control subjects from those attending the clinic for global control of cardiovascular risk at CCM. The demographic and clinical features of the two study groups are listed in Table 1 . The subjects were assessed with detailed medical history, physical examination, and echocardiography. In all patients, blood collection was performed before coronary angiography and surgery, with the exception of controls, which underwent samples collection at a scheduled visit.

\subsection{Blood Sampling and Biochemical Measurements}

Whole Blood. Peripheral blood sample was drawn from patients and controls while fasting into tubes containing EDTA (9.3 mM; Vacutainer Systems, Becton Dickinson, Franklin Lakes, NJ, USA) kept on ice and immediately precipitated with $10 \%$ trichloroacetic acid (Sigma-Aldrich, St. Louis, MO, USA) in $1 \mathrm{mM}$ EDTA solution. After centrifugation at $10,000 \mathrm{~g}$ for $10 \mathrm{~min}$ at $4^{\circ} \mathrm{C}$, the supernatant was stored at $-80^{\circ} \mathrm{C}$ until analysis.

Plasma. EDTA anticoagulated blood was centrifuged at $3,000 \mathrm{~g}$ for $10 \mathrm{~min}$ at $4^{\circ} \mathrm{C}$ within $30 \mathrm{~min}$ after being drawn. 
Plasma was separated and aliquots were stored at $-80^{\circ} \mathrm{C}$ until analysis.

2.3. Oxidative Stress Measurement. For oxidative stress evaluation whole blood concentrations of the oxidized (GSSG) and reduced (GSH) form of glutathione, whose ratio (GSSG/GSH) is a well-recognized index, were assessed by a previously developed and validated LC-MS/MS method [15].

2.4. Osteoprotegerin Evaluation. Plasma levels of soluble OPG were measured with an enzyme-linked immunosorbent assay (ELISA) kit (DuoSet, R\&D) following manufacturer instructions. The standard of this particular kit is similar to full-length OPG, making this ELISA kit more representative of circulating OPG molecule [9].

2.5. Statistical Analysis. Continuous variables are summarized as mean \pm standard error, except for age, which is represented as mean [minimum, maximum], while categorical variables are summarized as frequency and percentage.

For data analysis, Mann-Whitney test has been performed between MVP and control classes on continuous variables, while Fisher's exact test has been performed on discrete ones.

Finally, taking into account OPG measurement and GSSG/GSH ratio, we have implemented several logistic regression procedures in order to identify a classification rule, able to predict outcomes with the highest accuracy.

The logistic model is

$$
p\left(Y_{i}=\text { “MVP" }\right)=\frac{1}{1+e^{-\left(\beta_{0}+\beta_{1} x_{1}+\beta_{2} x_{2}\right)}},
$$

where $p$ is probability to have MVP, $x_{1}$ is OPG measurement (in $\mathrm{pg} / \mathrm{mL}$ ), and $x_{2}$ is GSSG/GSH ratio.

A receiver operating characteristic (ROC) curve has been plotted for each model and performances have been evaluated by comparing areas under the ROC Curve (AUC).

\section{Results}

3.1. Patient Characteristics. In this study we analysed 43 patients and 29 controls matched by age, sex, diabetes, hypertension, hypercholesterolemia, and smoking habits. The two groups were comparable for all the clinical and demographic features considered, except for BMI, which was significantly lower in MVP patients than in controls ( $p=0.005)$, and as expected, New York Heart Association (NYHA) class that was significantly higher in MVP patients $(p<0.001$, Table 1$)$. Drug therapies were not significantly different between MVP and controls, apart from beta-blockers, mostly taken by patients. However, up to now, no data are available about any influence of this drug class on oxidative stress levels. In Table 2 the qualitative and quantitative echocardiographic characteristics of mitral valves are reported. As expected the peak $E$ velocity and the ratio between peak $E$ and peak $A$ velocity $(E / A$ ratio) were significantly different between the two groups $(p<0.001)$.
TABLE 2: Echocardiography evaluation.

\begin{tabular}{lccc}
\hline $\begin{array}{l}\text { Echocardiography } \\
\text { parameters }\end{array}$ & Control $(N=29)$ & MVP $(N=43)$ & $p$ value \\
\hline LVEF $(\%)$ & $65.9 \pm 1.6$ & $63 \pm 1.6$ & 0.416 \\
Peak $E$ velocity $(\mathrm{cm} / \mathrm{s})$ & $72.8 \pm 3.8$ & $103 \pm 4.2$ & $<\mathbf{0 . 0 0 1}$ \\
Deceleration $E(\mathrm{~ms})$ & $229 \pm 13.4$ & $199 \pm 7.7$ & 0.094 \\
Peak $A$ velocity $(\mathrm{cm} / \mathrm{s})$ & $79.1 \pm 4.8$ & $68.9 \pm 2.8$ & 0.111 \\
E/A ratio & $0.96 \pm 0.07$ & $1.6 \pm 0.1$ & $<\mathbf{0 . 0 0 1}$ \\
EROA $\left(\mathrm{cm}^{2}\right)$ & - & $0.6 \pm 0.04$ & \\
\hline
\end{tabular}

LVEF: left ventricular ejection fraction; EROA: effective regurgitant orifice area. Mean \pm standard error.

3.2. Osteoprotegerin Levels and Oxidative Stress Status. The assessment of OPG levels revealed that this protein was significantly higher in MVP patients when compared to controls ( $1748 \pm 100.2$ versus $1109 \pm 45.3 \mathrm{pg} / \mathrm{mL}$, respectively; $p<0.0001$, Figure 1(a)). Since it has already been shown that OPG concentration increases with age [9], we implemented an adjusted model for this variable and the difference between the groups maintains its significance $(p<0.0001)$. In addition, we adjusted for NYHA class as well as BMI and the difference in OPG levels remained significant between the two groups $(p<0.01)$. Finally, we confirmed that the oxidative stress status, represented by GSSG/GSH ratio, was higher in MVP patients compared to control subjects $(0.116 \pm 0.007$ versus $0.053 \pm 0.013$, respectively; $p<0.0001$, Figure 1(b)) and remained significant even after NYHA class and BMI adjustment $(p<0.01)$. Notice that no correlation has been found between NYHA class and OPG levels or GSSG/GSH ratio.

3.3. Binary Logistic Regression Model. To assess if OPG or GSSG/GSH ratio could be used as potential circulating markers of MVP, we implemented a step-wise binary logistic regression model. To further improve the specificity and sensibility, we took OPG and GSSG/GSH ratio alone and then together. We also evaluated their possible interaction; however, it was not statistically significant $(p=0.243)$ and therefore we did not include it in the regression model. The regression parameters obtained from estimation procedure were $\beta_{0}=-7.590, \beta_{1}=0.005$, and $\beta_{2}=24.493$. The logistic model implemented is described in Section 2.5. This model was able to correctly classify 62 samples out of 72 (Figure 2). In addition, the logistic regression model pointed out an odds ratio of 38.5 (95\% CI: 9.9-150.6; $p<0.0001)$ to have MVP for subjects with $p\left(Y_{i}=\right.$ "MVP" $)>0.5$.

3.4. Combination of Osteoprotegerin and Oxidative Stress as Potential Circulating Marker of MVP. Receiver operator characteristic curve (ROC) was performed to determine if OPG or GSSG/GSH ratio alone or together could be used to identify MVP patients. As shown in Figure 3, OPG and GSSG/GSH ratio had an area under ROC curve (AUC) of 0.83 and 0.79 , respectively $(p<0.0001)$. However, if we considered OPG combined with GSSG/GSH we observed 




(a)



(b)

FIGURE 1: Osteoprotegerin and oxidative stress levels. (a) Osteoprotegerin (OPG) enzyme-linked immunosorbent assay (ELISA) on plasma samples from control subjects (CTRL) and mitral valve prolapse (MVP) patients. (b) Ratio between oxidized (GSSG) and reduced (GSH) form of glutathione as oxidative stress status index.

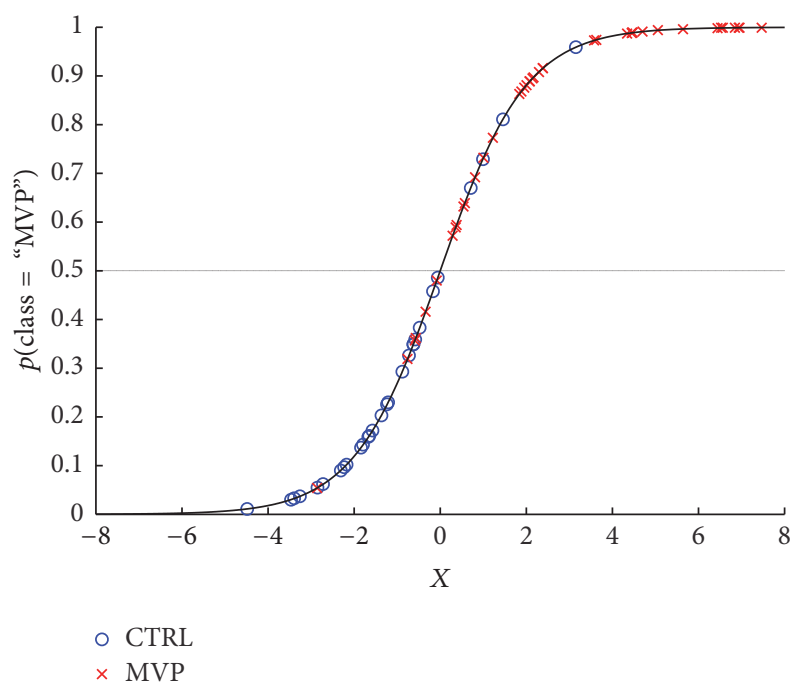

FIGURE 2: Binary logistic regression model. Graph representing the prediction of the best binary logistic regression model.

accuracy in terms of AUC of 0.92 with 95\% CI: $0.86-0.98$ and $p<0.0001$ (red thick line; Figure 3).

\section{Discussion}

Myxomatous mitral valve prolapse (MVP) with severe regurgitation (MR) is the most common cause for mitral valve surgery. The MVP diagnosis is some of the most challenging

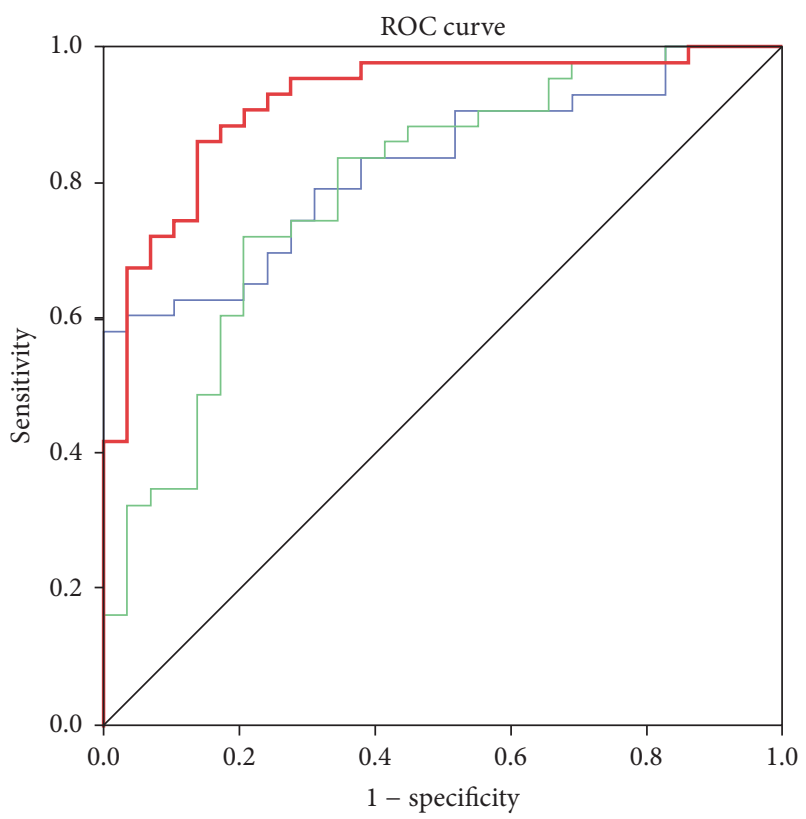

AUC

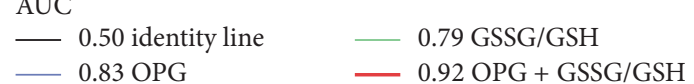

FIGURE 3: Osteoprotegerin combined with oxidative stress, as potential circulating marker of MVP. Receiver operating characteristic (ROC) curves and area under the curve (AUC) of Osteoprotegerin (OPG), ratio between oxidized (GSSG) and reduced (GSH) form of glutathione (GSSG/GSH) and their combination (OPG + GSSG/GSH). (Control subjects $n=29$, Mitral Valve Prolapse patients $n=43 ; p<0.0001$ ). 
aspects in clinical cardiology and, up to now, echocardiography is the only clinical reliable tool. No biomarkers are available for this pathology and, to the best of our knowledge, only a comparative proteomic study on plasma samples from 24 pooled MVP patients with moderate to severe MR revealed reduced levels of haptoglobin, platelet basic protein, and complement component C4b in the MVP/MR patients as compared to the 24 pooled matched control cases [16]. However, the clinical applicability is unclear, in part because most of the identified biomarkers had low AUC. In addition, Thalji et al. [12] and Sainger et al. [17] identified novel players that could be involved in myxomatous mitral valve degeneration and their data offer novel insights into the pathogenesis of MVP but no circulating biomarkers have been evaluated.

Results of the present work show that circulating OPG and oxidative stress status are positively associated with severe MR due to MVP. Based on these premises, we developed a multivariable logistic regression model with OPG and GSSG/GSH levels. This model is able to correctly identify $86 \%$ of MVP patients and $86 \%$ of control subjects.

Since all patients, in the MVP group, were the ones who underwent surgery due to symptoms, we evaluated and excluded that left ventricular ejection fraction (LVEF) and heart failure (NYHA classes) were the causes that increased OPG plasma levels and oxidative stress status. Therefore, our data supports that severe MR caused by myxomatous MVP could be identify through a multivariable binary logistic regression model.

The analyses of OPG as well as oxidative stress alone are not disease specific; indeed OPG has already been linked to coronary artery syndromes [18], aortic valve stenosis [19], myocardial infarction [20], and cardiovascular postoperative outcome [21]. However, the combination of these biochemical parameters allowed us to identify these patients with high accuracy.

Recently, we showed that OPG was involved in mitral valve endothelial to mesenchymal transition and plasma OPG levels permitted to identify MVP patients with an AUC of 0.92 [8]. However, all the selected MVP patients had only posterior MVP. It is known that two-thirds of MVP patients present the posterior prolapse, while the remaining third show anterior or bileaflet prolapse $[22,23]$. Considering this, to obtain a model able to discriminate MVP from healthy subjects, in primary screening, we must use a population with not only posterior but also anterior and bileaflet prolapse. Thus, in the present study we enrolled 43 MVP patients: 28 with posterior MVP (65\%) and 15 with anterior or bileaflet MVP (35\%). In this population, OPG had an AUC of 0.83 that was brought back to 0.92 when OPG was combined with GSSG/GSH ratio; meaning that OPG alone is not sufficient to discriminate with high accuracy the MVP mixed population (posterior, anterior, and bileaflet) from healthy subjects.

The present study has some limitations: first, the exiguous number of patients enrolled and the lack of strong diseasespecificity of OPG and oxidative stress with MVP. In addition, in our cohorts we had only two cases with mitral annulus calcification (MAC). Since OPG is involved in many cellular processes, in particular calcification, further studies need to address also the possible implication of this molecule not only in patients with MAC but also in those who have mitral valve stenosis. Lastly, patients with severe regurgitation compose the MVP cohort and further studies are needed to evaluate if this model is able to discriminate mild or moderate mitral valve regurgitation in the presence or absence of prolapse.

\section{Conclusion}

In conclusion, considering the prevalence of this disease (over $6 \% \geq 65$ years old [1]), the aging population, and the price of the echocardiographic evaluation, the health system costs may rise if no pharmacological treatment or cheaper diagnosis tools will be identified. In particular, circulating molecular signatures could be used in primary screening to identify possible MVP patients and then a confirmatory echocardiography could be performed only in these subjects. Thus, the study of new circulating markers and the combination of already known ones involved in MVP progression could lead to novel insights and possibly new therapeutic targets. To the best of our knowledge, this is the first study to show a strong association between OPG and oxidative stress status. In addition, these molecules could be measured in the clinical setting by the implementation and validation of diagnostic enzyme-linked immunosorbent assay (ELISA) for OPG and colorimetric assay for GSH and GSSG. Finally, since it is quite hard to believe that one single protein could discriminate two populations with high specificity and sensitivity, we believe that this approach could improve the identification of several signatures not only in mitral valve disease. However, the mechanisms explaining these correlations are still unclear; further molecular studies along with clinical validations will be necessary to confirm our findings.

\section{Abbreviations \\ AUC: Area under the curve \\ BMI: Body-mass index \\ GSH: Reduced glutathione \\ GSSG: Oxidized glutathione \\ HDL: High-density lipoproteins \\ MAC: Mitral annulus calcification \\ MVP: Mitral valve prolapse \\ OPG: Osteoprotegerin.}

\section{Competing Interests}

The authors declare that they have no competing interests.

\section{Authors' Contributions}

Paola Songia and Benedetta Porro equally contributed to this work.

\section{Acknowledgments}

The authors thank the entire Cardiac Surgery Unit at Centro Cardiologico Monzino for clinical data and blood collection. 
This work was supported by the Fondazione Gigi e Pupa Ferrari ONLUS and the Italian Ministry of Health [RC2015BIO30-2613051 to Centro Cardiologico Monzino].

\section{References}

[1] L. A. Freed, D. Levy, R. A. Levine et al., "Prevalence and clinical outcome of mitral-valve prolapse," New England Journal of Medicine, vol. 341, no. 1, pp. 1-7, 1999.

[2] S. Vahanian A, O. Alfieri, F. Andreotti et al., "Guidelines on the management of valvular heart disease (version 2012)," European Heart Journal, vol. 33, no. 19, pp. 2451-2496, 2012.

[3] F. N. Delling and R. S. Vasan, "Epidemiology and pathophysiology of mitral valve prolapse: new insights into disease progression, genetics, and molecular basis," Circulation, vol. 129, no. 21, pp. 2158-2170, 2014.

[4] R. G. Singh, R. Cappucci, R. Kramer-Fox et al., "Severe mitral regurgitation due to mitral valve prolapse: risk factors for development, progression, and need for mitral valve surgery," American Journal of Cardiology, vol. 85, no. 2, pp. 193-198, 2000.

[5] C. Deroyer, J. Magne, M. Moonen et al., "New biomarkers for primary mitral regurgitation," Clinical Proteomics, vol. 12, article 25, 2015.

[6] V. Cavalca, E. Tremoli, B. Porro et al., "Oxidative stress and nitric oxide pathway in adult patients who are candidates for cardiac surgery: patterns and differences," Interactive Cardiovascular and Thoracic Surgery, vol. 17, no. 6, pp. 923-930, 2013.

[7] J.-Y. Kim, Y.-J. Park, K.-J. Kim, J.-J. Choi, W.-U. Kim, and C.-S. Cho, "Osteoprotegerin causes apoptosis of endothelial progenitor cells by induction of oxidative stress," Arthritis and Rheumatism, vol. 65, no. 8, pp. 2172-2182, 2013.

[8] P. Songia, E. Branchetti, A. Parolari et al., "Mitral valve endothelial cells secrete osteoprotegerin during endothelial mesenchymal transition," Journal of Molecular and Cellular Cardiology, vol. 98, pp. 48-57, 2016.

[9] C. Pérez De Ciriza, A. Lawrie, and N. Varo, "Osteoprotegerin in cardiometabolic disorders," International Journal of Endocrinology, vol. 2015, Article ID 564934, 15 pages, 2015.

[10] A. Avignon, A. Sultan, C. Piot et al., "Osteoprotegerin: a novel independent marker for silent myocardial ischemia in asymptomatic diabetic patients," Diabetes Care, vol. 30, no. 11, pp. 2934-2939, 2007.

[11] D. Gordin, A. Soro-Paavonen, M. C. Thomas et al., "Osteoprotegerin is an independent predictor of vascular events in finnish adults with type 1 diabetes," Diabetes Care, vol. 36, no. 7, pp. 1827-1833, 2013.

[12] N. M. Thalji, M. A. Hagler, H. Zhang et al., "Nonbiased molecular screening identifies novel molecular regulators of fibrogenic and proliferative signaling in myxomatous mitral valve disease," Circulation: Cardiovascular Genetics, vol. 8, no. 3, pp. 516-528, 2015.

[13] M. A. Hagler, T. M. Hadley, H. Zhang et al., "TGF- $\beta$ signalling and reactive oxygen species drive fibrosis and matrix remodelling in myxomatous mitral valves," Cardiovascular Research, vol. 99, no. 1, pp. 175-184, 2013.

[14] K. Thirunavukkarasu, R. R. Miles, D. L. Halladay et al., "Stimulation of osteoprotegerin (OPG) gene expression by transforming growth factor- $\beta$ (TGF- $\beta$ ). Mapping of the OPG promoter region that mediates TGF- $\beta$ effects," Journal of Biological Chemistry, vol. 276, no. 39, pp. 36241-36250, 2001.
[15] I. Squellerio, D. Caruso, B. Porro, F. Veglia, E. Tremoli, and V. Cavalca, "Direct glutathione quantification in human blood by LC-MS/MS: comparison with HPLC with electrochemical detection," Journal of Pharmaceutical and Biomedical Analysis, vol. 71, pp. 111-118, 2012.

[16] H. T. Tan, L. H. Ling, M. C. Dolor-Torres, J. W.-L. Yip, A. M. Richards, and M. C. M. Chung, "Proteomics discovery of biomarkers for mitral regurgitation caused by mitral valve prolapse," Journal of Proteomics, vol. 94, pp. 337-345, 2013.

[17] R. Sainger, J. B. Grau, E. Branchetti et al., "Human myxomatous mitral valve prolapse: role of bone morphogenetic protein 4 in valvular interstitial cell activation," Journal of Cellular Physiology, vol. 227, no. 6, pp. 2595-2604, 2012.

[18] D. Tousoulis, G. Siasos, K. Maniatis et al., "Serum osteoprotegerin and osteopontin levels are associated with arterial stiffness and the presence and severity of coronary artery disease," International Journal of Cardiology, vol. 167, no. 5, pp. 1924-1928, 2013.

[19] A. Borowiec, R. Dąbrowski, I. Kowalik et al., "Osteoprotegerin in patients with degenerative aortic stenosis and preserved left-ventricular ejection fraction," Journal of Cardiovascular Medicine, vol. 16, no. 6, pp. 444-450, 2015.

[20] A. Margonato, R. Gorla, A. Macchi et al., "Role of plaque calcification regulators osteoprotegerin and matrix Gla-proteins in stable angina and acute myocardial infarction," Journal of Cardiovascular Medicine, vol. 16, no. 3, pp. 156-162, 2015.

[21] S. M. Venuraju, A. Yerramasu, R. Corder, and A. Lahiri, "Osteoprotegerin as a predictor of coronary artery disease and cardiovascular mortality and morbidity," Journal of the American College of Cardiology, vol. 55, no. 19, pp. 2049-2061, 2010.

[22] D. R. Johnston, A. M. Gillinov, E. H. Blackstone et al., "Surgical repair of posterior mitral valve prolapse: implications for guidelines and percutaneous repair," The Annals of Thoracic Surgery, vol. 89, no. 5, pp. 1385-1394, 2010.

[23] A. E. Weyman and M. Scherrer-Crosbie, "Marfan syndrome and mitral valve prolapse," The Journal of Clinical Investigation, vol. 114, no. 11, pp. 1543-1546, 2004. 


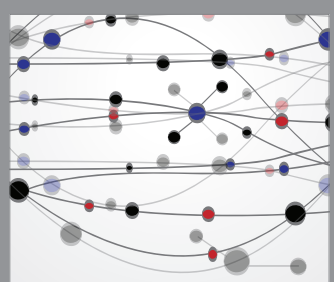

The Scientific World Journal
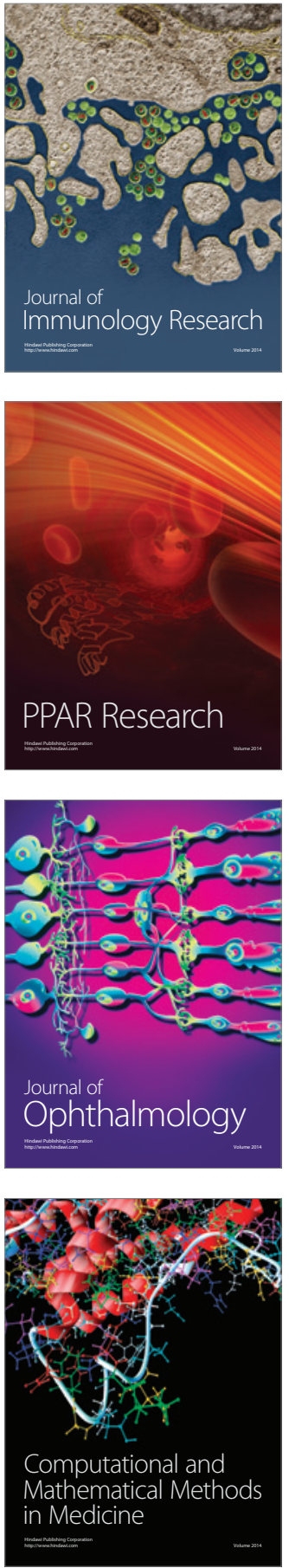

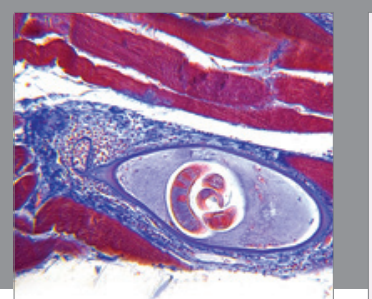

Gastroenterology Research and Practice
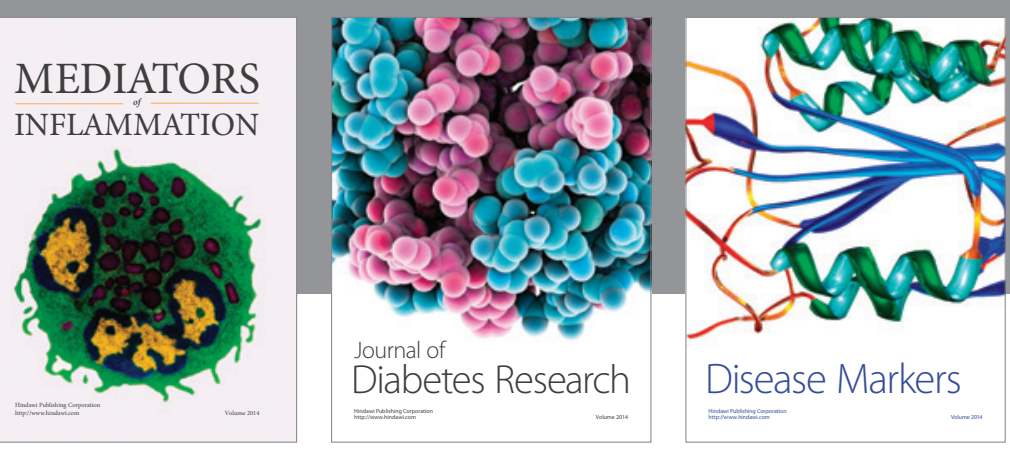

Disease Markers

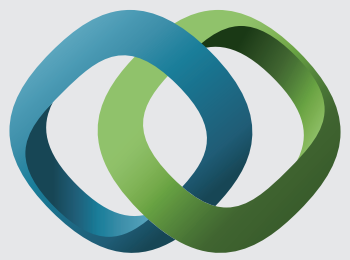

\section{Hindawi}

Submit your manuscripts at

https://www.hindawi.com
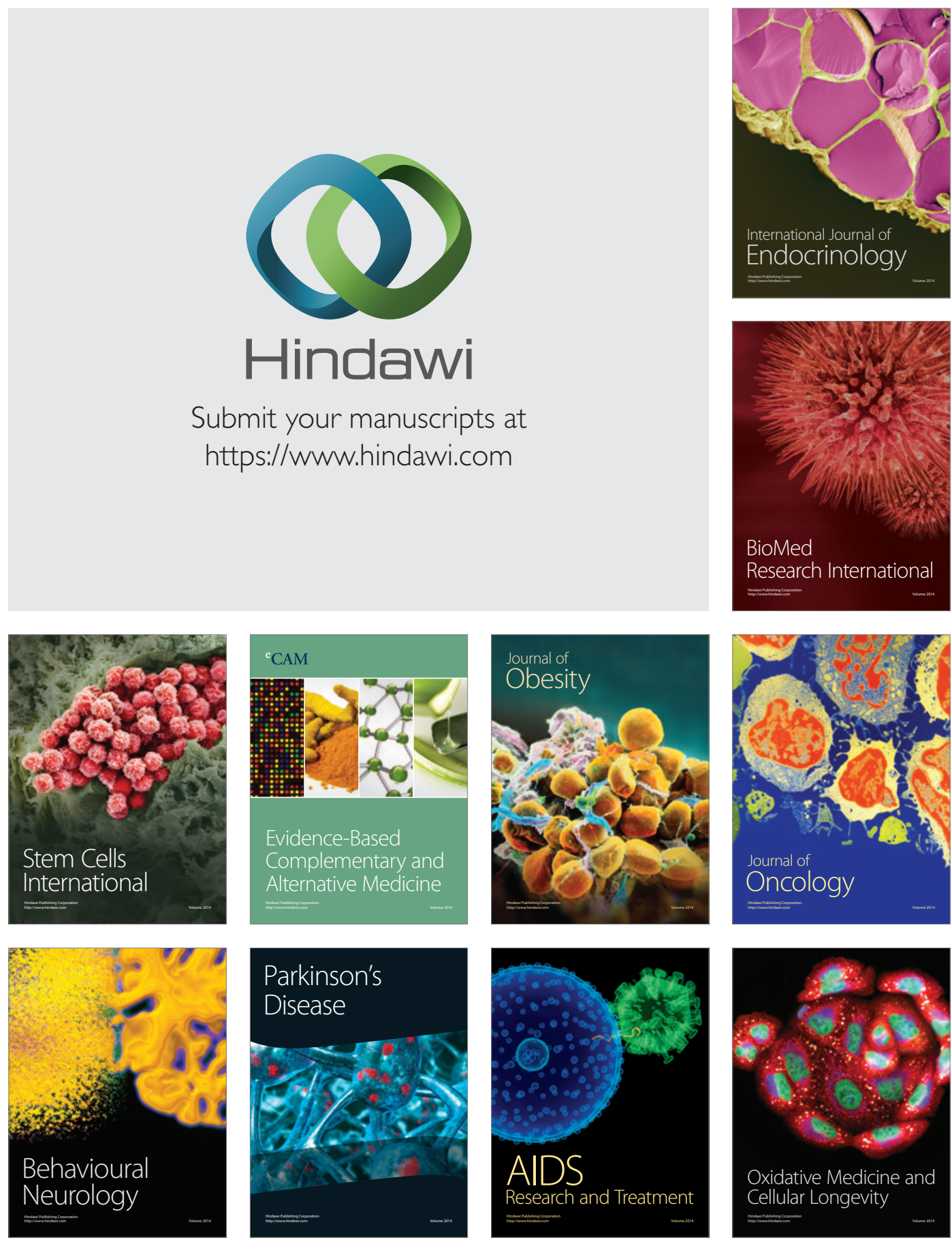